\title{
Variation in Compliance In Two Classes of Two-Link Underactuated Mechanisms
}

\author{
Ravi Balasubramanian and Aaron M. Dollar
}

\begin{abstract}
The compliance of an underactuated robotic hand, or a robotic hand with fewer actuators than degrees of freedom, is a function of the mechanism type, the design parameters, and the operational control mode. The transmissions used in underactuated mechanisms can be divided into two main classes based on the selfadaptive transmission used to route actuation to the various degrees of freedom, namely the single-acting transmission and the double-acting transmission. While both transmission classes can be represented using a kinematic constraint equation that defines the relationship between actuator and joint motion, the main difference between the two transmission classes is that the kinematic constraint is always active in double-acting mechanisms while there are specific combinations of external disturbances and mechanism parmeters that render the constraint inactive in single-acting mechanisms. While previous studies have only explored the performance of underactuated mechanisms with the constraint always active, this paper identifies the benefits for robotic grasping (such as better disturbance rejection) that arise when the constraint becomes inactive in single-acting mechanisms.
\end{abstract}

\section{INTRODUCTION}

A strong interest in improving the reliability of robotic grasping has led to the development of "underactuated" robotic hands, or hands with fewer actuators than degrees of freedom. These hands exploit clever transmission mechanisms that couple the motion of multiple joints to an actuator while allowing the hands to naturally adapt to the external environment without any sensing. While the motion of these hands when actuated internally has been studied in prior work [2], [11], [9], [21], [23], the compliance exhibited by these hands in the presence of external disturbances is still not well understood. This paper investigates the variation in compliance of an underactuated mechanism as

This work was supported in part by the National Science Foundation grant IIS-0953856. R. Balasubramanian (contact author) and A. M. Dollar are with the Department of Mechanical Engineering at Yale University, New Haven, CT, USA. \{ravi.balasubramanian, aaron.dollar\}@yale.edu. a function of the mechanism type, design parameters, and external loading.

A robotic hand's compliance is an important factor in grasping performance since it influences how the hand adapts to the environment and disturbance forces. Compliance in robotic hands is typically included in hand design in the form of passive torsional springs at the robot's joints or through active control such as jointtorque control policies [24], [12]. In the case of underactuated hands, an additional factor that determines hand compliance is the transmission mechanism linking actuator and joint motion.

The transmission mechanisms used in current underactuated hand designs can be categorized into two broad classes: single-acting mechanisms and doubleacting mechanisms. Single-acting mechanisms can apply only unidirectional forces on the joints. For example, Fig. 1a shows a cable-driven system which produces flexion (curling) motion through a pulling action, and the return motion is achieved using springs. Examples of robotic hands with single-acting mechanisms include the SDM Hand [9], Balance Bar [14], and $100 \mathrm{G}$ robotic hands [15].

On the other hand, a double-acting mechanism can apply bi-directional forces on the joints such as a pull and a push (see Fig 1c for a linkage-driven example). Examples of robotic hands with double-acting mechanisms include the Laval hands [10], [17], SPRING [3], Southampton [7], Graspar [6], BarrettHand [27], and Obrero [26] robotic hands (see [1] for other examples of underactuated mechanisms).

Interestingly, the coupling behavior of mechanisms used in single-acting and double-acting classes may be represented by the same kinematic constraint that defines the relationship between actuator and joint motion (see section II-B for more details). However, a significant difference between the two classes is that there are certain operating modes and external loads for which the coupling constraint becomes inactive for a single-acting mechanism (for example, when the cable slackens in a single-acting mechanism, see 


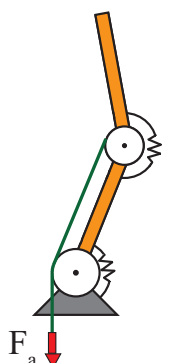

(a)

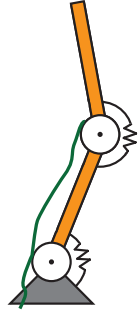

(b)

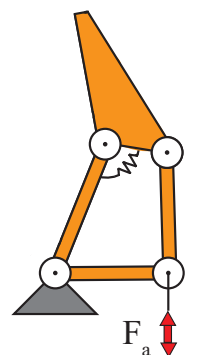

(c)
Fig. 1. Examples of underactuated hands: (a) single-acting cabledriven system, (b) single-acting when coupling breaks down (cable slack), and (c) double-acting linkage-driven system.

Fig. 1b) while the coupling constraint is always active for a double-acting mechanism. To our knowledge, prior work [16], [25] has only studied the behavior of underactuated mechanisms when the constraint is always active, thus, missing an important difference between the two mechanism classes.

Building on preliminary work of how an underactuated mechanism changes posture due to external disturbances at a specific configuration and specific parameter values [1], we quantify in this paper how an underactuated mechanism's compliance varies as a function of the coupling mechanism's behavior in the presence of external disturbances, by specifically identifying the parameter subspace in which the coupling constraint becomes inactive in single-acting mechanisms. Furthermore, we analyze the effect of different control modes, namely position control mode or force control mode, on mechanism compliance. In section II, we present a framework for studying the compliance of an underactuated mechanism taking into consideration the kinematics of joint coupling, hand joint configuration, joint stiffness, force location, and the control mode. In section III, we present results from an analysis of the compliance of single-acting and double-acting underactuated hands. Finally, in section IV, we present a discussion of the effect of compliance variation on grasping performance and then offer suggestions for the design and operation of underactuated hands.

\section{FrameWORK FOR Underactuated HAND ANALYSIS}

The framework for analyzing underactuated hands consists of three components: 1) The static equilibrium equations that relate contact forces on the phalanxes with joint torques; 2) The kinematic coupling between joint motion and actuator motion; 3) The change in

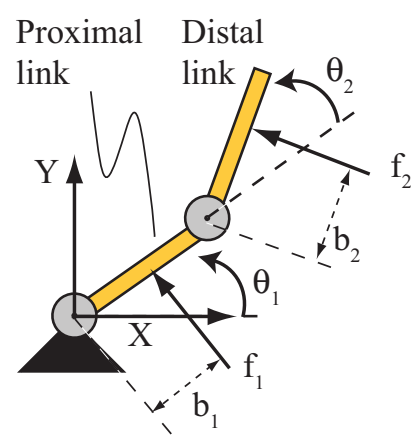

Fig. 2. A two-link revolute-revolute finger. Examples of actuation mechanisms are shown in Fig. 1.

robot configuration due to the external forces in the presence of joint coupling. The configuration change of the mechanism for a given force is used to compute the mechanism compliance. As an example, we consider the flexion and extension behavior of a twolink revolute-revolute finger with a single actuator (see Fig. 2).

\section{A. Static Equilibrium}

The relationship between the contact forces acting on the finger and the resulting joint torques (see Fig. 2) may be expressed as

$$
\tau=J_{c}^{T} f_{e},
$$

where $f_{e}=\left(\begin{array}{c}f_{1} \\ f_{2}\end{array}\right)$ represents the normal contact force on the proximal and distal links, $\tau=\left(\begin{array}{l}\tau_{1} \\ \tau_{2}\end{array}\right)$ the resulting torque at the joints, and $J_{c} \in \mathbb{R}^{2 \times 2}$ the contact Jacobian that maps between the two spaces. For a two-link mechanism, the contact Jacobian $J_{c}$ can be computed as [2]

$$
J_{c}=\left(\begin{array}{cc}
b_{1} & 0 \\
b_{2}+l_{1} \cos \theta_{2} & b_{2}
\end{array}\right),
$$

where $b_{1}$ represents the proximal force location, $b_{2}$ the distal force location, $l_{1}$ the proximal link length, $\theta_{2}$ the relative angle between the two links. While this formulation assumes that the contact point can slide on the link without friction, friction models can also be incorporated.

\section{B. Kinematics of the Coupling Mechanism}

The kinematics of the coupling mechanism may be expressed as a first-order differential equation in the mechanism's configuration $\theta=\left(\begin{array}{c}\theta_{1} \\ \theta_{2}\end{array}\right)$ and actuator 
variable $\theta_{a}$. In cable-driven mechanisms, the actuator variable may be defined as the angle traveled by the pulley over which the cable travels, while in linkage driven mechanisms, the actuator variable may be defined as the angle traveled by the actuating link.

For cable-driven mechanisms in current underactuated hands such as the SDM hand, the kinematics of the coupling mechanism may be expressed as

$$
\Delta \theta_{a}=r_{1} \Delta \theta_{1}+r_{2} \Delta \theta_{2},
$$

where $r_{1}$ and $r_{2}$ represent the pulley radii (assuming unit radius for the actuation pulley) and $\Delta \theta_{i}$ the configuration change of link $i$.

For four-bar linkage driven mechanisms in current underactuated hands such as the SARAH hand, the kinematics of the coupling mechanism may be expressed as

$$
\Delta \theta_{a}=\Delta \theta_{1}+\Delta \theta_{2} R,
$$

where $R$ represents the transmission ratio of the mechanism. Note that for a four-bar linkage mechanism, the transmission ratio $R$ is a function of joint configuration $\theta$ and link lengths and therefore varies with joint motion. In this paper, we consider only small joint configuration changes from a given configuration $\theta$. Thus, $R$ is a constant for the instantaneous analysis in this paper.

A closer analysis of (3) and (4) shows that the kinematics of both cable-driven mechanisms and linkagedriven mechanisms can be expressed as

$$
J_{a} \Delta \theta=\Delta \theta_{a},
$$

where $J_{a}=\left(\begin{array}{ll}a_{1} & a_{2}\end{array}\right)$ represents the actuator Jacobian of the mechanism. For linkage-driven systems, $a_{1}$ equals 1 and $a_{2}$ equals $R$. For cable-driven systems, $a_{1}$ equals $r_{1}$ and $a_{2}$ equals $r_{2}$. For both types of systems, the transmission ratio may be defined as $R=a_{2} / a_{1}$. Note that some underactuated hands with double-acting mechanisms use clutch or break mechanisms and are not compliant (see [27] for an example). We do not explore such hands in this paper.

\section{Robot Configuration Change Due to External Force}

An external disturbance force $f_{e}$ on an underactuated hand can cause a change in configuration $\Delta \theta$. The magnitude and direction of $\Delta \theta$ in the joint configuration space depends on factors such as 1) the joint coupling mechanism, 2) the direction, magnitude, and location of the disturbance force, 3) joint compliance, and 4) the hand control mode. This section describes the change in robot configuration as a function of all four factors (assuming disturbance forces normal to the links).

The configuration change $\Delta \theta$ for external force $f_{e}$ can be quantified using a Lagrangian view of the work done by the external forces and the energy stored in the springs in the presence of the actuation constraints [13]. Specifically, we define the Lagrangian $L$ as

$$
L=W_{s}+W_{c}+W_{a}
$$

where $W_{s}$ represents the work done on the springs, $W_{c}$ the work done by the external forces, and $W_{a}$ the work done on the actuator. The work done on the spring $W_{s}=-1 / 2 \Delta \theta^{T} K \Delta \theta$ and work done by the external forces $W_{c}=f_{e}^{T} J_{c} \Delta \theta$ [5] are similar in form for the underactuated mechanisms we consider, and $K=\left(\begin{array}{cc}K_{1} & 0 \\ 0 & K_{2}\end{array}\right)$ represents joint stiffness.

However, the work done on the actuator $W_{a}$ takes different forms depending on the control mode the mechanism is driven in (see Table I, which also gives the scenarios where these control modes apply). In the force-control mode, the work is "real", while in the position control mode, the "virtual" work must equal zero [19]. In the decoupled mode (constraint inactive), the work done on the actuator is zero (see Fig. 1b). The formulation in this paper assumes that the external force is constant through the deflection. Certainly, cases where the external force changes with deflection (such as compliant springy contacts) as well as geometric constraints that would be imposed by contact can also be incorporated. In position control mode, $p$ is the pretension in the actuating mechanism that maintains mechanism stability prior to the application of the external disturbance $f_{e}$. The structure of the contact forces have been intentionally kept simple so as to focus on the hand configuration change.

By taking derivatives of the Lagrangian with respect to the variables and any Lagrange multipliers, we can derive the static balance equations [13]. Table I presents the static balance equations for the two-link mechanism, one for each control mode. Note that these equations predict the instantaneous hand posture change $\Delta \theta$ from a statically stable configuration as a result of the external force and the actuation mode.

\section{Mechanism Compliance}

A mechanism's compliance at a point may be defined as the net deflection of that point on the mechanism due to a unit force. The effective mechanism compliance in the presence of multiple external forces has been derived in [20] for fully-actuated robotic arms, but has 
TABLE I

EFFECT OF CONTROL MODE ON WORK DONE ON ACTUATOR BY EXTERNAL FORCES

\begin{tabular}{l|l|c|l} 
Actuation mode & $\begin{array}{l}\text { Work done on } \\
\text { actuator }\end{array}$ & Static balance equation & Example scenario \\
\hline $\begin{array}{l}\text { Force control (con- } \\
\text { stant actuator force) }\end{array}$ & $W_{a}=f_{a} \Delta \theta_{a}^{1}$ & $K \Delta \theta+J_{c}^{T} f_{e}+J_{a}^{T} f_{a}=0 \quad$ (7) & $\begin{array}{l}\text { Maintaining fixed cable tension in } \\
\text { SDM hand. }\end{array}$ \\
$\begin{array}{l}\text { Position control (con- } \\
\text { stant actuator posi- } \\
\text { tion) }\end{array}$ & $\begin{array}{l}\text { Virtual work } \\
W_{a}=\lambda \Delta \theta_{a}=0^{2}\end{array}$ & $\begin{array}{c}J_{a} \Delta \theta \\
=0+J_{c}^{T} f_{e}+J_{a}^{T}(\lambda-p)=0 \\
\text { 1) Maintaining fixed cable length in } \\
\text { SDM hand (can save battery power } \\
\text { if actuator can be locked); 2) Non- } \\
\text { backdrivability in SARAH hand. }\end{array}$ \\
$\begin{array}{l}\text { Decoupled (coupling } \\
\text { constraint inactive) }\end{array}$ & $W_{a}=0$ & $K \Delta \theta+J_{c}^{T} f_{e}=0(9)$ & Cable slackening in SDM hand. \\
\hline \hline
\end{tabular}

${ }^{1}: f_{a}$ is the constant actuation force. ${ }^{2}: \lambda$ is the tendon force resulting from the coupling constraint.

not yet been well understood for underactuated hands. For simplicity, we consider the case where a normal external force is applied only at the distal link of an underactuated mechanism. This situation is similar to the circumstances of a precision grasp [18] where only the distal link interacts with an object. Large external disturbances are also most likely to occur on the distal link as it is more likely to make contact with objects in the external environment.

Since a coordinate frame attached to the mechanism at the force location has three degrees of freedom in the plane (translations parallel and perpendicular to the link and a rotational degree of freedom), the mechanism compliance at that point for a normal distal force is three-dimensional. The three elements represent the compliance perpendicular to direction of the normal force (tangent to the link), the compliance in the direction of the normal force, and the torsional compliance. In this paper, we only focus on mechanism compliance in the direction of the normal force.

For a distal link force $f_{2}$, the mechanism compliance $C$ can be computed as

$$
C=\Delta d / f_{2},
$$

where $\Delta d \in \mathbb{R}$ represents the deviation of the contact point in the force's direction $v=\left(\begin{array}{c}\mathrm{s}_{12} \\ -\mathrm{c}_{12}\end{array}\right), \mathrm{s}_{12}=$ $\sin \left(\theta_{1}+\theta_{2}\right)$, and $c_{12}=\cos \left(\theta_{1}+\theta_{2}\right)$.

Note that we can use the differential kinematics of the two-link mechanism to compute the mapping from joint configuration change $\Delta \theta$ to the change in location of the contact point $\Delta x \in \mathbb{R}^{2}$ as follows:

$$
\Delta x=J_{k} \Delta \theta
$$

where $J_{k} \in \mathbb{R}^{2 \times 2}$ is the manipulator kinematic Jacobian [5]. For a two-link mechanism, the Jacobian $J_{k}$ for a contact point on the distal link may be expressed as

$$
J_{k}=\left(\begin{array}{cc}
-l_{1} \mathrm{~s}_{1}-b_{2} \mathrm{~s}_{12} & -b_{2} \mathrm{~s}_{12} \\
l_{1} \mathrm{c}_{1}+b_{2} \mathrm{c}_{12} & b_{2} \mathrm{c}_{12}
\end{array}\right),
$$

where $\mathrm{s}_{i}=\sin \theta_{i}$ and $\mathrm{c}_{i}=\cos \theta_{i}$. The deviation $\Delta d$ in the direction of the force may be computed as $\Delta d=\Delta x \cdot v$.

A closer look at static balance in the decoupled mode (see (9) in Table I) reveals that the compliance at the force location is determined primarily by the joint stiffnesses $K_{1}$ and $K_{2}$ and the force location. The system compliance in force-control mode also (see (7) in Table I) is shaped by the joint stiffnesses $K_{1}$ and $K_{2}$ and force location only, even though the actuator maintains a constant force. This is because the constant actuation force $f_{a}$ only produces a constant shift in joint configuration which is not a function of the external force. Thus, the constant actuator force does not affect mechanism compliance in force-control mode. In the force control mode and the decoupled mode, the joint coupling does not influence mechanism compliance.

In contrast, the system response in the position control mode (see (8) in Table I) is shaped by the joint compliance $K_{1}$ and $K_{2}$, the pulley radii ratio $R$ (due to the kinematic constraint created by the fixed actuator position), and the mechanism pretension $p$. Indeed, the pretension value $p$ influences the circumstances under which the coupling mechanism in single-acting systems transitions into the decoupled mode (coupling constraint inactive). For example, external forces can combine to cause the cable to go slack, and the single-acting mechanism transitions into the decoupled control mode. Since the underactuated mechanism exhibits more interesting compliance behavior in position control mode when compared with the compliance behavior in force control or the decoupled mode, the 
TABLE II

FIXED PARAMETERS

\begin{tabular}{c|c}
\hline \hline Parameter & Value \\
\hline Proximal joint stiffness $K_{1}$ & $1 \mathrm{Nm} / \mathrm{rad}$ \\
Proximal and distal link length $l_{1}$ and $l_{2}$ & $0.1 \mathrm{~m}$ \\
Proximal pulley radius $r_{1}$ & $0.02 \mathrm{~m}$ \\
Proximal joint configuration $\theta_{1}$ & $\pi / 10 \mathrm{rad}$ \\
Cable pretension $p$ & $10 \mathrm{~N}$ \\
External force on proximal link $f_{1}$ & $0 \mathrm{~N}$ \\
\hline \hline
\end{tabular}

rest of this paper only highlights the results from the position control mode.

\section{RESULTS}

Using the SDM hand [9] as an exemplar of a cabledriven single-acting underactuated mechanism and the SARAH hand [17] as a linkage-driven double-acting underactuated mechanism, we now present results from a kinetostatic analysis of the variation in mechanism compliance using the static stability models from section II. We examine the variation in compliance over a nominal set of the following parameter space: the joint stiffness ratio $K_{r}=K_{2} / K_{1}$, transmission ratio $R$, distal link configuration $\theta_{2}$, and distal force location $b_{2}$. Table II shows the values of the fixed parameters used in the analysis. We show results for only two distal-joint configurations that are nominally seen in the grasping process. Since the static balance of a two-link finger is a continuous system, it is straightforward to work out performance at other joint configurations.

\section{A. Compliance of a Double-Acting Mechanism}

Fig. 3 shows the variation in compliance of a linkagedriven double-acting mechanism in position control mode. We now analyze the variation in compliance across the rows and columns of the subfigures to study the effect of the joint stiffness ratio $K_{r}$ and the distal joint configuration $\theta_{2}$. As expected, as the joint stiffness ratio increases from $K_{r}=0.5$ to $K_{r}=10$, the overall compliance decreases.

We also notice that the mechanism has zero compliance for some combination of parameters. This point has been termed an "equilibrium point" in prior work [2], [22], [1]. In the context of the studying a mechanism's compliance, we term this point a "zerocompliance point" instead and use the same terminology in the rest of the paper. At the zero-compliance point, the mechanism behaves extremely stiff and can withstand large external forces without any deviation. Using our framework, we can analyze the variation of the zero-compliance point across the complete parameter space for underactuated hands. It is interesting to note that the zero-compliance point whose expression has the form $e=l_{1} R \cos \theta_{2} /(1-R)$ does not vary with the joint stiffness ratio $K_{r}$, since it is dependent only on the transmission ratio $R$, distal joint configuration $\theta_{2}$, and proximal link length $l_{1}$.

As the distal joint configuration $\theta_{2}$ increases from $\theta_{2}=\pi / 6$ to $\theta_{2}=\pi / 3$, the compliance also decreases. This is expected because the moment arm of a normal force on the distal link about the proximal joint decreases as the distal joint angle $\theta_{2}$ increases. An interesting aspect not shown in these plots is that the zero-compliance curve shifts rapidly towards the $b_{2}=0$ and $R=1$ locations as the distal link approaches the perpendicular configuration $\left(\theta_{2}=\pi / 2\right)$. This indicates that at large flexion angles the mechanism exhibits large stiffness only when the normal disturbance force is very close to the distal joint or when the pulley radius ratio $R$ is close to unity. The condition $R=1$ is particularly interesting, since in this situation the proximal joint motion is exactly opposite to the distal joint motion, causing the distal joint angle to be fixed relative to a world coordinate frame.

We now look closely at each subfigure to study the effect of the pulley radius ratio $R$ and the distal force location $b_{2}$ on the effective compliance. As expected, the compliance $C$ increases as the distal force moves farther away from the distal joint.

The effect of the pulley-radius ratio on mechanism compliance is more intricate. First, we notice that for nominal distal link configurations $\left(0<\theta_{2}<\pi / 2\right)$, the mechanism exhibits zero compliance only when $R<1$. Also, we can carefully choose $R$ to locate the zerocompliance point beyond the link length if we ignore large flexion angles ( $\theta_{2}$ between 84 and 90 degrees).

\section{B. Compliance of a Single-Acting Mechanism}

For extension disturbance forces, a single-acting mechanism like the SDM hand in position control mode behaves identical to the double-acting mechanism since the coupling constraint is always active (cable tension always couples the proximal and distal joint motion; see Fig. 3). However, a single-acting mechanism in position control mode exhibits bimodal compliance for flexion disturbance force (see Fig. 4), depending on if the cable is slack or taut. Indeed, a large enough flexion force can cause the cable to go slack, rendering the coupling constraint inactive.

In Fig. 4, the thin (red) lines in the left region represent the compliance contours when the joints are 

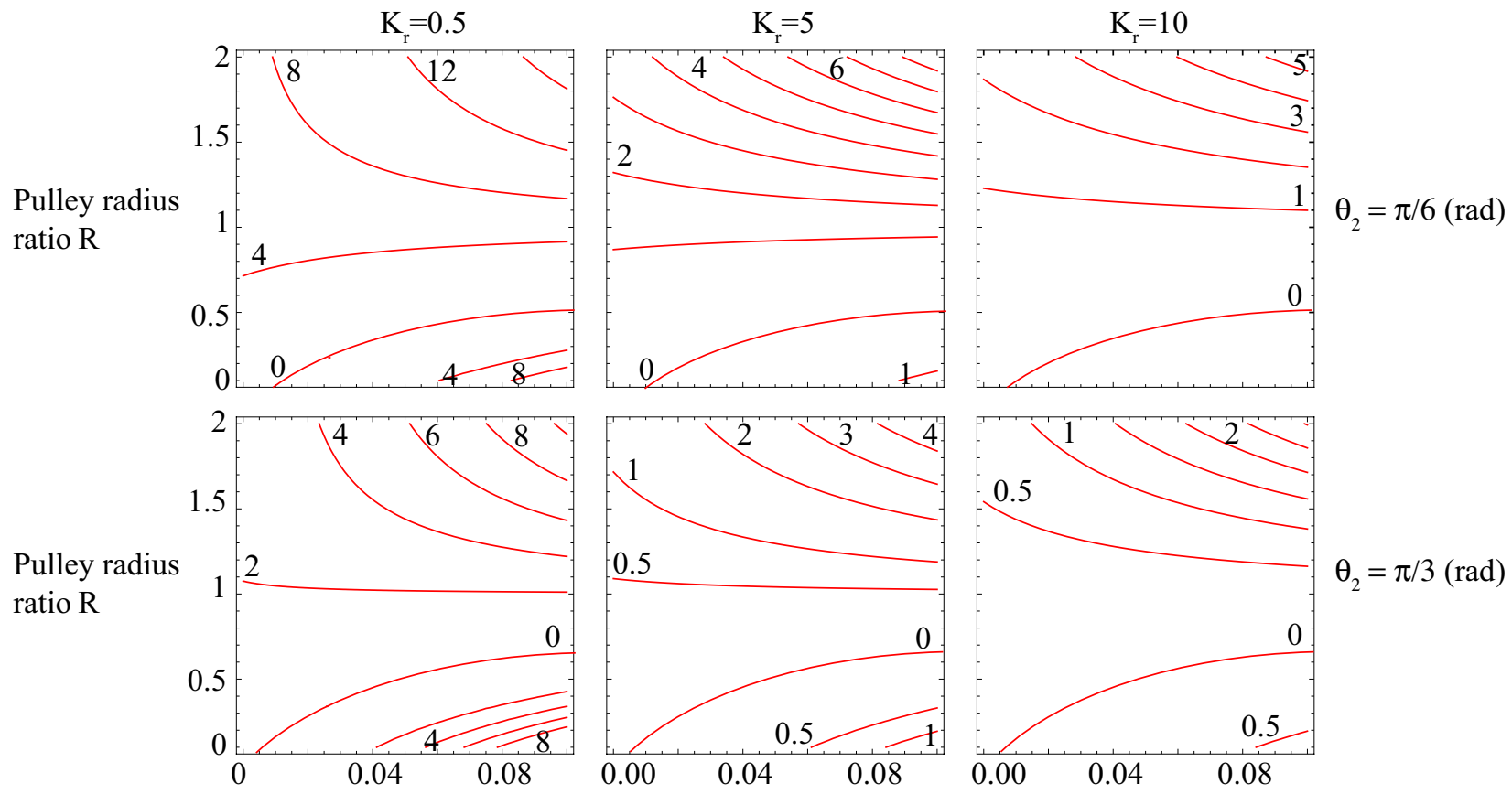

Force location $b_{2}(m)$

Fig. 3. Variation of the compliance $(\mathrm{mm} / \mathrm{N})$ of an underactuated mechanism with an active coupling constraint (such as in a double-acting mechanism). The parameters explored are distal link configuration $\theta_{2}$, joint stiffness ratio $K_{r}$, the pulley radius ratio $R$, and distal force location $b_{2}$. The joint stiffness ratio $K_{r}$ increases from left to right across the subfigures and the joint angle $\theta_{2}$ increases from top to bottom across the subfigures.
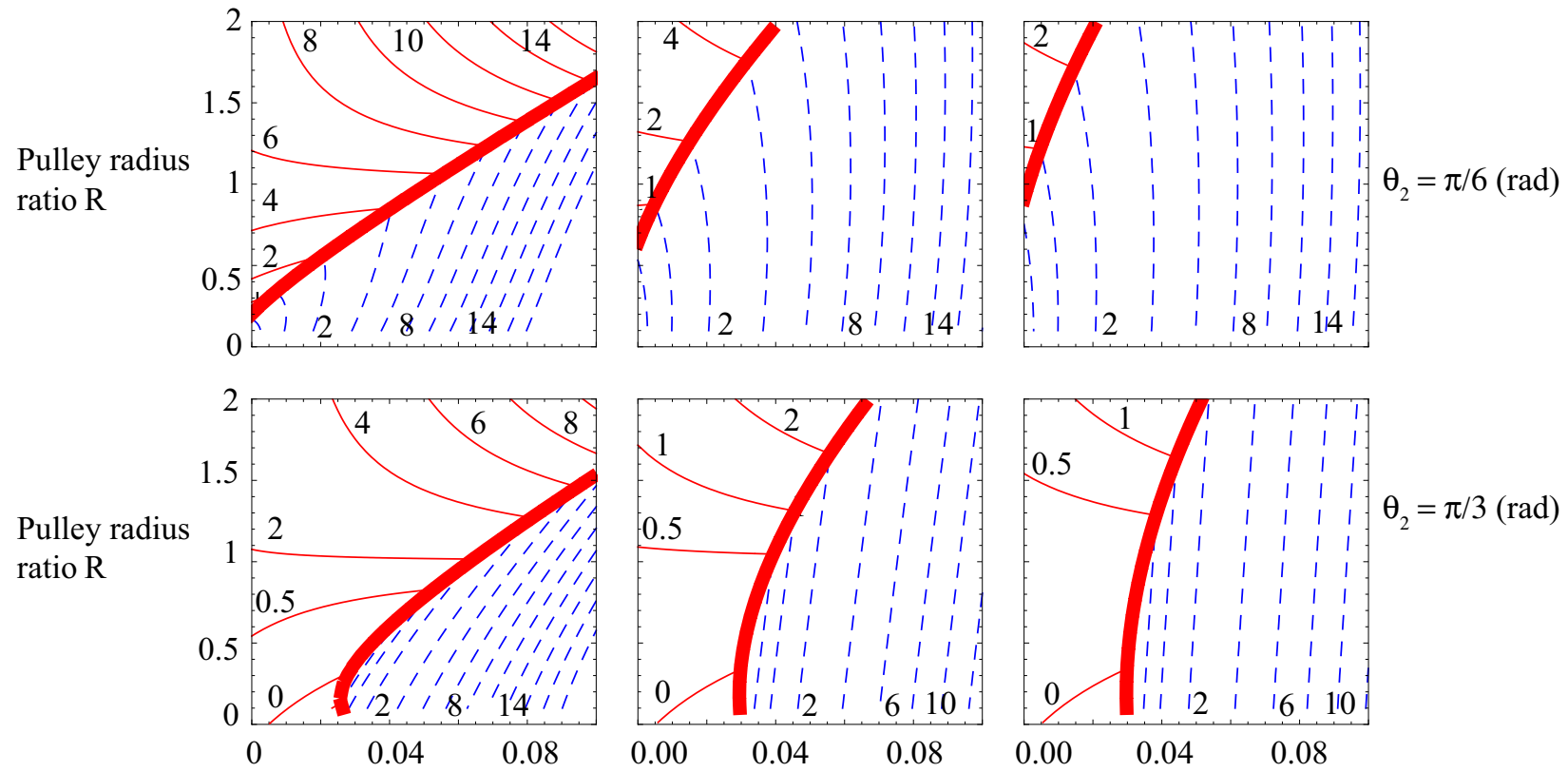

Force location $b_{2}(m)$

Fig. 4. Variation of the compliance $(\mathrm{mm} / \mathrm{N})$ of an underactuated mechanism where the coupling constraint can become inactive (such as in a cable-driven single-acting mechanism in position control mode). The parameters explored are distal link configuration $\theta_{2}$, joint stiffness ratio $K_{r}$, pulley radius ratio $R$, and distal force location $b_{2}$. The joint stiffness ratio $K_{r}$ increases from left to right across the subfigures and the joint angle $\theta_{2}$ increases from top to bottom across the subfigures. The thick (red) solid line represents the parameter combinations at which the mechanism transitions into the decoupled mode. The thin (red) lines represent parameter combinations at which the joints are coupled, and the blue (dotted) lines represent parameter combinations at which the joints are completely decoupled. 
still coupled, and the compliance behavior of the singleacting mechanism is identical to the compliance of a double-acting mechanism in this region. However, certain parameter combinations (indicated by the solid red line) can render the coupling constraint inactive, such as when the cable becomes slack in the cabledriven mechanism. The parameter space to the right of this line represents the region when the joints are completely decoupled. Note that the boundary between these regions is a function of the pretension $p$ in the system and shifts to the right as the pretension increases.

We notice that the compliance is generally higher in the decoupled region when compared with the region when the joints are coupled. This is expected since most of the stiffness for the underactuated mechanism in position control mode comes from the joint coupling. Also, the variation of mechanism compliance with pulley radius ratio $R$ in the decoupled state is much lower than in the coupled state. While one may have expected the decoupled compliance to be independent of pulley radius ratio $R$, the single-acting mechanism actually transitions from a constraint-active state to a constraint-inactive state. Thus, the effect of pulley radius ratio $R$ on compliance in the constraint-active state carries over to the effective compliance even in the constraint-inactive state.

\section{DISCUSSION}

\section{A. Bimodal Compliance in Single-Acting Mechanisms}

The key result in this paper is identifying the parameter space where the coupling constraint becomes inactive in single-acting mechanisms. The constraint becoming inactive in an underactuated mechanism does have benefits, as it provides a desirable disturbance rejection behavior [1]. Specifically, in a cable-driven single-acting mechanism like the SDM hand, the pretension $p$ in the cable determines when the coupling constraint becomes inactive due to a flexion force. When the coupling constraint becomes inactive, the mechanism yields to the external disturbance force by curling in naturally and possibly even strengthening a grasp by enveloping the object (see Fig. 5a). In contrast, a double-acting mechanism where the constraint is always active exhibits complex reconfiguration which can negatively influence a grasp (see Fig. 5b).

The parameter space where the coupling constraint becomes inactive increases for lower pretension values, suggesting that pretension should be kept as small as possible while retaining a stable grasp. Furthermore,

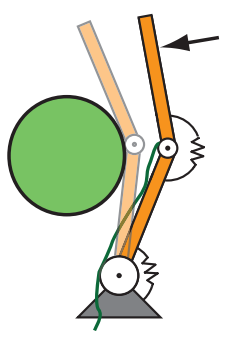

(a)

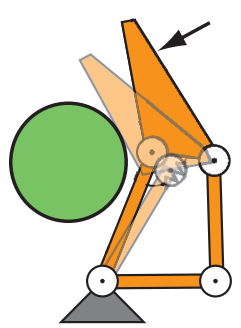

(b)
Fig. 5. Disturbance response in position-control mode of (a) a single-acting mechanism where the coupling constraint becomes inactive (cable slackens). The mechanism complies naturally with the disturbance force and curls in. (b) Disturbance response of a double-acting mechanism where the coupling constraint is always active. The mechanism exhibits complex reconfiguration where the distal joint flexes and the proximal joint extends.

Fig. 3 suggests that this parameter subspace also increases with larger joint-stiffness ratio $K_{r}$. This accords with previous results that showed that the distal joint should be much stiffer than the proximal joint in order to retain a desirable hand grasping configuration in the presence of object contact forces [8]. Independently, it has also been shown that a higher joint stiffness ratio $K_{r}$ helps in grasp stability as well [4]. Note that in this paper we have only considered two degree-of-freedom fingers and normal external forces on the distal links. More work is required to expand the parameter space to include contact constraints and fingers with more degrees of freedom.

\section{B. Effect of Zero-Compliance Point On Grasping}

From Figs. 3 and 4, we notice that there are regions in the parameter space where the mechanism has zero compliance; that is, at those points, the mechanism will not deviate for any external force normal to the link. So how do these zero-compliance points influence hand performance when used in a grasping task? When the hand approaches an object in the pre-grasp phase, any collisions with the object at or near the zero-compliance point will produce minimal finger reconfiguration and poor adaptability. In contrast, if the hand has already grasped an object, then an external force that acts at or close to the zero-compliance point will also produce minimal hand reconfiguration. Thus, the grasp will be rigid to external disturbances which is advantageous. More work is required to understand how the placement of zero-compliance points through hand design can influence grasping performance. 


\section{Tunable Stiffness with Variable Transmission Ratio}

For both single-acting and double-acting mechanisms, we notice that the transmission ratio $R$ influences mechanism compliance. In the case of double-acting mechanisms implemented using four-bar linkages, the transmission ratio $R$ is governed by joint configuration as well as link lengths. In the case of singleacting cable-driven systems, the transmission ratio $R$ is governed by pulley radii (or more generally, the moment arm of the tendon insertion point). It would be interesting to explore a design strategy in which the transmission ratio $R$ can be varied during the grasping process to achieve optimal compliance at the contact points. For example, once a grasp is achieved, the pulley radius ratio $R$ could be tuned in order to provide high stiffness at the contact points. For cable-driven systems, a cam with varying radius could be used as a pulley.

\section{ACKNOWLEDGMENT}

The authors thank Joseph Belter for helpful discussions on underactuated mechanisms and Lael Odhner for helpful discussions on the Lagrangian formulation.

\section{REFERENCES}

[1] R. Balasubramanian, J. T. Belter, and A. M. Dollar. External disturbances and coupling mechanisms in underactuated hands. In Proc. of ASME Internat. Design Engineering Technical Conf and Computers and Information in Engineering Conf., 2010.

[2] L. Birglen, T. Laliberté, and C. Gosselin. Underactuated Robotic Hands. Springer, 2008.

[3] M. C. Carrozza, C. Suppo, F. Sebastiani, B. MASSA, F. Vecchi, R. Lazzarini, M. R. Cutkosky, and P. Dario. The spring hand: Development of a self-adaptive prosthesis for restoring natural grasping. Autonomous Robots, pages 125-141, 2004.

[4] M. Ciocarlie and P. Allen. A design and analysis tool for underactuated compliant hands. In Proc. of the IEEE/RSJ Internat. Conf. on Intell. Robots and Sys., pages 5234-5239, 2009.

[5] J. J. Craig. Introduction to Robotics. Addison Wesley, 1989.

[6] J. D. Crisman, C. Kanojia, and I. Zeid. Graspar: A flexible, easily controllable robotic hand. IEEE Robotics and Automation Magazine, pages 32-38, 1996.

[7] R. M. Crowder, V. N. Dubey, P. H. Chappell, and D. R. Whatley. A multi-fingered end effector for unstructured environments. In Proc. IEEE Internat. Conf. on Robotics and Automation, pages 3038-3043, 1999.
[8] A. M. Dollar and R. D. Howe. Towards grasping in unstructured environments: Grasper compliance and configuration optimization. Adv. Robotics, 19(5):523-543, 2005.

[9] A. M. Dollar and R. D. Howe. A robust compliant grasper via shape deposition manufacturing. ASME/IEEE Trans. on Mechatron., 11(2):154-161, 2006.

[10] C. Gosselin and T. Laliberté. Underactuated mechanical finger with return actuation. US Patent 5762 390, 1996.

[11] S. Hirose and Y. Umetani. The development of soft gripper for the versatile robot hand. Mechanism and IVlechine Theory, 13:351-359, 1978.

[12] N. Hogan. Impedance control of a robotic manipulator. In Proc. of Winter Annual Meeting of ASME, 1981. DOI: $10.1017 / \mathrm{S} 0263574708004281$.

[13] T. Inoue and S. Hirai. Mechanics and Control of Soft-fingered Manipulation. Springer, 2008.

[14] Y. Kamikawa and T. Maeno. Underactuated five-finger prosthetic hand inspired by grasping force distribution of humans In Proc. IEEE/RSJ Internat. Conf. on Intell. Robots and Sys., pages 717-722, 2008.

[15] M. Kaneko, M. Higashimori, R. Takenaka, A. Namiki, and M. Ishikawa. The $100 \mathrm{G}$ capturing robot too fast to see. ASME/IEEE Trans. on Mechatronics, 8(1):37-44, 2003.

[16] G. A. Kragten and J. L. Herder. Equilibrium, stability, and robustness in underactuated grasping. In Proc. of ASME Internat. Design Engineering Technical Conf., pages 645-652, 2007.

[17] T. Laliberté and C. Gosselin. Actuation system for highly underactuated gripping mechanism. US Patent 6505870 , 2002.

[18] C. L. MacKenzie and T. Iberall. The grasping hand. Elsevier, 1994.

[19] R. M. Murray, Z. X. Li, and S. S. Sastry. A Mathematical Introduction to Robotic Manipulation. CRC Press, 1994.

[20] J. Park and O. Khatib. Robot multiple contact control. Robotica, 2008.

[21] M. Rakic. Multifingered hand with self-adaptability. Robotics and Computer-Integrated Manufact., 3(2):269-276, 1989.

[22] R. Rizk, S. Krut, and E. Dombre. Grasp-stability analysis of a two-phalanx isotropic underactuated finger. In Proc. IEEE/RSJ Internat. Conf. on Intell. Robots and Sys., pages 3289-3294, 2007.

[23] A. Rovetta. On functionality of a new mechanical hand. ASME J. Mechanical Design, 103:277-280, 1981.

[24] J. K. Salisbury. Active stiffness control of a manipulator in cartesian coordinates. In Proc. IEEE Conf. Decision and Control, 1980.

[25] J. Schuurmans, R. Q. van der Linde, D. H. Plettenburg, and F. C. van der Helm. Grasp force optimization in the design of an underactuated robotic hand. In Proc. of Internat. Conf. on Rehabilitation Robotics, pages 776-782, 2007.

[26] E. Torres-Jara. Obrero: A platform for sensitive manipulation. In Proc. IEEE-RAS Internat. Conf. on Humanoid Robots, pages 327-332, 2005.

[27] W. T. Townsend. The barretthand grasper-programmably flexible part handling and assembly. Industrial Robot: An International Journal, 27(3):181-188, 2000. 